\title{
Constitutive Model Research on Pure Aluminum Considering Dynamic Recovery and Recrystallization
}

\author{
Tingting Zhou ${ }^{1,}$, Yang Yang ${ }^{1, b}$ and Maobing Shuai ${ }^{1, c}$ \\ ${ }^{1}$ Science and Technology on Surface Physic and Chemistry Laboratory, Jiangyou 621908, China \\ a775943970@qq.com, b937815402@qq.com, c'shuaimaobing@caep.cn
}

Keywords: pure aluminum; constitutive model; dynamic recovery; recrystallization

Abstract. The article introduces some main constitutive models of pure aluminum with regard to mechanical response, dynamic recovery and recrystallization during the process of severe plastic deformation. Two kinds of constitutive models will be introduced in this paper, because there are few models considering two aspects simultaneously, that is, material deformation dynamics and microstructure evolution. In the case of deformation dynamics, the JC model, the ZA model, the power exponent function model will be introduced in detail; in view of microstructure evolution, the M. Zhou explicit dynamic constitutive model, a model considering dynamic recrystallization will be introduced in detail. At last, this paper will present the BCJ constitutive model specially, which has superiority at macro level and micro level, and has been widely used abroad. An analysis of the characteristics of these models, and scope of application of each model, comparison between each model as well as the common problems of these models will be summarized. Furthermore, the article will exhibit some characteristics of pure aluminum and the reasons for selecting the BCJ constitutive model to describe the deformation mechanisms of pure aluminum during single point diamond cutting.

\section{Introduction}

Pure aluminum exhibits the characteristics of high plasticity and low hardness. It's easily subjected to intense deformation during single point diamond cutting. It belongs to high-fault-energy FCC metal. It tends to occur easily cross slip or climb during deformation, followed by dynamic recovery [1]. Moreover, when the deformation parameters are adjusted appropriately, its plastic deformation capacity can be greatly increased, followed by dynamic recrystallization [2]. Therefore, it's important to establish a dynamic constitutive relation which can reflect the dynamic recovery and recrystallization of pure aluminum during the deformation. This work will be of great significance for the mechanism study of material deformation and prediction of material deformation trends.

At present, the constitutive model, describing the material flow stress, can be roughly divided into two categories. One kind of models are using mathematical methods or are based on physics in a certain extent. They are called empirical or semi-empirical models, e.g. the Johnson-Cook model, the power exponent function model and the Zerilli-Armstrong model. This kind of models are simple in form and have been widely applied. Another kind of models are physics-based, which consider the microstructure evolution, such as the $\mathrm{M}$. Zhou explicit dynamic constitutive model, the model considering the dynamic recrystallization and the BCJ model etc. This kind of models involve some information such as dislocation proliferation or annihilation, static or dynamic recovery and recrystallization in the deformation process, which is helpful to study the deformation mechanisms for the materials.

\section{The factors considered in constitutive models}

Constitutive equation is a mechanical response rule, reflecting the mathematical relationship between flow stress and other factors during deformation process. The factors, influencing the rheological stress, are strain, strain rate, temperature and other load conditions. They belong to the external causes; Other factors (e.g. material composition, grain size, deformation activation energy and the 
microstructure of material) belong to the internal causes. The two kinds of factors influence the magnitude and change trends of flow stress by strain hardening and temperature softening, static or dynamic recovery as well as static or dynamic recrystallization[3]. Now, however, there are few constitutive models can fully include the influences of the external causes and internal causes, except several physics-based models, e.g. the BCJ model. The article will present representative constitutive models from literatures at home and abroad, used for pure aluminum or aluminum alloy.

\section{Representative constitutive models}

\section{Zerilli-Armstrong constitutive model}

For BCC metal:

$\sigma=\mathrm{C}_{0}+\mathrm{C}_{1} \exp \left(-\mathrm{C}_{3} \mathrm{~T}+\mathrm{C}_{4} \mathrm{~T} \ln \xi\right)+\mathrm{C}_{5} \varepsilon^{\mathrm{n}}$.

For FCC metal:

$\sigma=\mathrm{C}_{0}+\mathrm{C}_{2} \varepsilon^{1 / 2} \exp \left(-\mathrm{C}_{3} \mathrm{~T}+\mathrm{C}_{4} \mathrm{~T} \ln \&\right.$.

where, the units of $\mathrm{C} 0, \mathrm{C} 1, \mathrm{C} 2$ and $\mathrm{C} 5$ are $\mathrm{MPa}$; for $\mathrm{C} 3, \mathrm{C} 4$, the units are $\mathrm{K}^{-1}$; n denotes a material constant; T denotes absolute temperature, whose unit is K[4]. As can be seen from the formula (1) and (2), for BCC metal, the dependence of the flow stress on the strain is not affected by the changes of temperature and strain rate, however the influence mechanism of the FCC materials is opposite.

Zerilli-Armstrong (Z-A) constitutive model is a semi-empirical constitutive model based on the dislocation, which can be used to describe armco iron, copper and aluminum in elastic-plastic deformation. It is fit for a wide range of temperature and strain rate, and has two forms to adapt to metals with different lattice structures [5-7]. But the Z-A model is lacking in the mechanism reflecting static or dynamic response, loading paths, and the effect of strain rate history. Furthermore, it's unable to capture the adiabatic effect [8-10].

\section{Johnson-Cook constitutive equation}

$$
\bar{\sigma}=\left(\mathrm{A}+\mathrm{B} \varepsilon^{\mathrm{n}}\right)(1+\mathrm{Cln} \delta)\left(1-\left(\frac{\mathrm{T}-\mathrm{T}_{\text {room }}}{\mathrm{T}_{\text {melt }}-\mathrm{T}_{\text {room }}}\right)^{\mathrm{m}}\right) .
$$

where, $\mathrm{T}, \mathrm{T}_{\text {room }}, \mathrm{T}_{\text {melt }}$ are deformation temperature, room temperature and material melting point temperature respectively; A is the material yield stress (unit: $\mathrm{MPa}$ ); $\mathrm{B}$ is the strain hardening constant (unit: MPa); C, n, $\mathrm{m}$ are the coefficients on material properties $[11,12]$.

The Johnson-Cook ( J-C) constitutive equation is simple, used widely to represent the constitutive relation of steel, aluminum and their alloys. It's applicable to describe the stress-strain relation of ferrous and non-ferrous metals under large strain rate [6]. But it is unable to reveal the physical basis of deformation. In addition, the effects of the strain, strain rate and temperature on stress are mutually coupled, the equation also lacks description of elastic deformation [5-7]. Furthermore, the relationship between stress and the logarithm of strain rate, which is seen as a linear relationship, is wrong in many cases [13].

\section{Power-exponent function model}

$$
\sigma=A \xi^{\mathrm{m}} \mathrm{e}^{-\mathrm{bT}} \text {. }
$$

where, A, m, b are material constants; $\mathrm{T}$ is the absolute temperature [14]. This model is applicable to describe rheological properties of high plastic material, such as pure aluminum. But it cannot consider the relationship between stress and strain, and the sensitivity of the high temperature is not very well. Some scholars, based on MARC platform[15], have carried out the secondary development, considering all materials as perfecting solid or fluid, as a result, the model gets subsection correction according to the temperature. But it still cannot make up for the effect of strain on the stress and cannot capture the adiabatic effect. 


\section{Zhou explicit dynamic recover model}

$$
\begin{aligned}
& \sigma=f_{1}(\varepsilon, \&, T) f_{2}(\&, T) . \\
& \sigma_{s}=f_{2}(\& \& T)=C \sinh ^{-1}\left[B\left(Z_{\varepsilon}\right)^{m}\right] . \\
& Z_{\varepsilon}=\& \exp \left(\frac{Q}{k T}\right) . \\
& f_{1}(\varepsilon, \& \& T)\left\{\begin{array}{l}
=0(\varepsilon=0) \\
=1\left(\varepsilon=\varepsilon_{s}\right)
\end{array}\right) \\
& \text { when } 0<\varepsilon<\varepsilon_{s}: \\
& f_{1}(\varepsilon, \&, T)=\left(1-\exp \left(-b \varepsilon^{n}\right)\right) .
\end{aligned}
$$

where, $f_{1}$ describes the strain-hardening area of the flow stress curve; $f_{2}$ describes the situation under the large deformation condition, that is, the flow stress will tend to be stable. And $f_{2}$ can be expressed by the hyperbolic function. For $f_{1}$, there are two conditions, as shown in equation (7). $\varepsilon_{s}$ is saturated strain. In the strain-hardening stage, $f_{1}$ can be represented as equation (9). $\mathrm{b}$ and $\mathrm{n}$ are related to $\mathrm{Z}$, which is temperature compensation strain rate[16].

The model contains the strain hardening effect, strain rate hardening effect and temperature softening effect. Introducing the deformation activation energy, it can capture the dynamic response process, and can explain the reasons of stress softening, that is, recovery and temperature. This model is simple, easy to use. But the model is not fit for simulate tension and compression experiment data[16].

\section{Recrystallization model}

There are a lot of models to simulate the static and dynamic recrystallization. Based on physical mechanism, DRX model can predict the evolution of the macroscopic stress and grain size, with the help of the auxiliary of multiphase plasticity and grain growth models, it can also describe quantitatively typical characteristics of dynamic recrystallization, such as unimodal form turning to multimodal form; steady-state convergence of stress and grain size; the energy relationship between stress and grain size, etc. But this model cannot predict stress softening phenomenon under high strain rate, cannot contain the influence of solid solution and the precipitated phases. Using the data of pure copper, which has a lot of commonness with pure aluminum, to find that the fitting curve can reflect the natural development process converging to a steady state of stress and strain, but the curve coincidence degree is not high[17]. S.P. CHEN et al.[18] proposed a recrystallization-kinetics model based on physics. The model takes the effect on recrystallization kinetics into account. The simulation of single-phase alloy AA1050 shows that nucleation density is closely related to the local strain and stress, however, the dependence of the annealing temperature is weak.

A lot of models, focusing on the simulation of the recrystallization behavior, involve basically the grain nucleation model, the growth model, and reflect material microstructure change, but the description of the macro flow stress is not specific, even lacking.

\section{Conclusions on representative constitutive models}

In fact, a variety of deformation mechanisms often coupled during the deformation process, the individual description for a deformation mechanism of one kind of constitutive models can't be suitable for complex rheological behaviors, making the application of the constitutive equation limited.

This paper will detail another constitutive model -- BCJ model, which is not widely used at home, but it have got many researches abroad. The applicability of model is wide. It can be adapted to high temperature, large strain and strain rate of deformation, and can also capture the deformation 
mechanisms of low temperature and low strain rate. At the same time, it is able to consider the macro and micro aspects. The BCJ model is good at simulating the rheological stress during the process of metal cutting and has great advantage on other types of material and other deformation processes.

\section{BCJ constitutive model}

The Bammann-Chiesa-Johnson constitutive model(BCJ model) proposed in 1984[19], is one of the few models considering the yield surface theory. This model is a unification of macro and micro constitutive model. After years of research and development, its theoretical maturity and application range have been improved greatly. In application, its simulation objects are from metal materials to the ceramic materials, and even geological materials (such as Bammann et al. (1995), Harley, E.J and Bammann (1999), Regueiro and Bammann (2002), Chuzhoy, et al. (2003), Solanki et al. (2007), Tucker et al. (2010), Solanki et al. (2010 a), Salehghaffari, S et al. (2011), Salehghaffari, S et al. (2012), Koffi Enakoutsa et al. (2012)). Because there are too many model parameters needing to be determined (up to 18 constants), the studies on fitting parameters of the BCJ model have been promoted (such as Guo, et al. (2005), M.F. Horstemeyer, (2009), Salehghaffari, S et al. (2011), Salehghaffari(2012), Guosheng Wang (2011), Tan Yang(2015) etc.).BCJ constitutive equations are as follows:

$$
\begin{aligned}
& \underline{\underline{\sigma}^{\circ}}=\underline{\underline{\alpha}}-\underline{\mathrm{w}^{e}} \underline{\underline{\sigma}}+\underline{\underline{\sigma}} \underline{\mathrm{w}^{e}}=\lambda \operatorname{tr}\left(\underline{D^{e}}\right) \underline{I}+2 \mu \underline{D^{e}} . \\
& \underline{D^{i n}}=f(T) \sinh \left(\frac{\|\underline{\sigma}-\underline{\alpha}\|-\{R+Y(T)\}}{V(T)}\right) \frac{\underline{\sigma}-\underline{\alpha}}{\|\underline{\underline{\alpha}}-\underline{\alpha}\|} . \\
& \underline{\alpha^{\circ}}=h(T) \underline{D^{i n}}-\left(\sqrt{\frac{2}{3}} r_{d}(T)\left\|\underline{D^{i n}}\right\|+r_{s}(T)\right) \underline{\alpha} \| \underline{\alpha} . \\
& \hat{\alpha}=H(T) \underline{D^{i n}}-\left(\sqrt{\frac{2}{3}} R_{d}(T)\left\|\underline{D^{i n}}\right\|+R_{s}(T)\right) R^{2} .
\end{aligned}
$$

The above equations are based on the theory of the basic law of thermodynamics. The strain of any point in material can be regarded as the sum of elastic strain and inelastic strain. Material mechanics performances are decided by the isotropic hardening variable $\mathrm{R}$ and kinematic hardening state variable $\alpha$, and these two variables are considered as completely independent. Formula (10), (11), (12) and (13) denote the linear elastic constitutive relation, flow rule, the evolution of kinematic hardening internal variable and the evolution of the isotropic hardening variable respectively, equation (12) and (13) are based on the evolution of the dislocation dynamics.

The BCJ model can not only describe the dynamic mechanical behavior under isothermal conditions, but also describe the material deformation considering the condition of adiabatic temperature rise. There are 9 scalar equations covering yield, recovery and hardening as seen in table $1[10,20]$.

Table 1. 9 scalar equations

\begin{tabular}{|l|l|l|}
\hline $\mathrm{V}(\mathrm{T})=\mathrm{C} 1 \exp (-\mathrm{C} 2 / \mathrm{T})$ & $\mathrm{r}_{\mathrm{d}}(\mathrm{T})=\mathrm{C} 7 \exp (-\mathrm{C} 8 / \mathrm{T})$ & $\mathrm{R}_{\mathrm{d}}(\mathrm{T})=\mathrm{C} 13 \exp (-\mathrm{C} 14 / \mathrm{T})$ \\
\hline $\mathrm{Y}(\mathrm{T})=\mathrm{C} 3 \exp (\mathrm{C} 4 / \mathrm{T})$ & $\mathrm{h}(\mathrm{T})=\mathrm{C} 9 \exp (\mathrm{C} 10 / \mathrm{T})$ & $\mathrm{H}(\mathrm{T})=\mathrm{C} 15 \exp (\mathrm{C} 16 / \mathrm{T})$ \\
\hline $\mathrm{f}(\mathrm{T})=\mathrm{C} 5 \exp (-\mathrm{C} 6 / \mathrm{T})$ & $\mathrm{r}_{\mathrm{s}}(\mathrm{T})=\mathrm{C} 11 \exp (-\mathrm{C} 12 / \mathrm{T})$ & $\mathrm{R}_{\mathrm{S}}(\mathrm{T})=\mathrm{C} 17 \exp (-\mathrm{C} 18 / \mathrm{T})$ \\
\hline
\end{tabular}

where, $\mathrm{V}(\mathrm{T}), \mathrm{Y}(\mathrm{T})$ and $f(\mathrm{~T})$ are temperature-dependence parameters of the Arrhenius type, associated with creep and the plasticity. $\mathrm{Y}(\mathrm{T})$ denotes the rate-independent yield stress, 
$f(\mathrm{~T})$ determines when the rate dependence affects initial yielding, $\mathrm{V}(\mathrm{T})$ determines the magnitude of rate dependence on yielding. $\mathrm{H}(\mathrm{T})$ and $h(\mathrm{~T})$ are the hardening moduli, $r_{d}(\mathrm{~T})$ and $\mathrm{R}_{\mathrm{d}}(\mathrm{T})$ describe the dynamic response, $r_{s}(\mathrm{~T})$ and $\mathrm{R}_{\mathrm{s}}(\mathrm{T})$ capture the static recovery and heat recovery. $\mathrm{Ci}(\mathrm{i}=1 \sim 18)$ is a material constant, which contains uncertainty reflecting indirectly the material microstructure changes. All 9 parameters are temperature-dependent, can capture the temperature history effect, and can also be used as the contact between material microstructure changes and the macroscopic mechanics.

The BCJ model covers both the macroscopic stress and microstructure, including dynamic mechanical response, static recovery, dynamic recovery and dynamic recrystallization. It can also capture the adiabatic temperature rise effect. The model retains the yield surface theory of the classical strength theory, whose fitting range is not limited to experimental data. In addition, BCJ model is suitable for low temperature and low strain rate deformation conditions, and can also be applied to high temperature and high strain rate deformation conditions. In summary, the BCJ model is fit for capturing and predicting intense deformationa at blade, the adiabatic temperature rise, the dynamic recovery and recrystallization during the cutting process of pure aluminum. Furthermore, it can also describe the influence of the Baushinger effect [21] on the dynamic mechanical properties of pure aluminum.

\section{Conclusions}

In this paper, the constitutive models applied to simulate pure aluminum or aluminum alloy are classified. After comparing the representative empirical, semi-empirical and physical models, the BCJ constitutive model is proposed, which is much more suitable for simulation of pure aluminum during single point diamond turning process. In summary, taking the characteristics and disadvantages of the above models, the processing technologies, the finite element simulation softwares and the development of large numerical calculation softwares into consideration, the future constitutive models will gradually tend to be more comprehensive, more functional and unified. The BCJ model responds to this trend exactly, and it undoubtedly has great prospects for the development of constitutive models. However, the parameter recognition method of the model is not sufficiently developed, and requires further exploration and adjustment.

\section{Acknowledgements}

This work was financially supported by NSAF of National Natural Science Foundation and China Academy of Engineering Physics (U153010011). The support of students in CAMLAB of Tsinghua University is appreciated.

\section{References}

[1]. YU, Y.N., Principles of Metal[M]. Metallurgical Industry Press, 2013.

[2].Wen, Q., Dynamic Mechanical Behavior. 2005, Alabama.

[3]. LIU, B.S., et al., A Review of Thermal Medium Forming Constitutive Modeling Method for Aluminum Alloy Sheet. Journal of Plasticity Engineering, 2011.

[4]. Zerilli, F.J. and R.W. Armstrong, Dislocation-Mechanics-Based Constitutive Relations for Material Dynamics Calculations. Journal of Applied Physics, 1987. 61(5): p. 1816

[5]. Samantaray, D., S. Mandal and A.K. Bhaduri, A Comparative Study on Johnson Cook, Modified Zerilli-Armstrong and Arrhenius-Type Constitutive Models to Predict Elevated Temperature Flow Behaviour in Modified 9Cr-1Mo Steel. Computational Materials Science, 2009. 47(2): p. 568-576. 
[6]. CHEN, D.N., et al., Some Problems in the Numerical Simulation of Plane Shock Wave of Ductile Materials. Journal of Solid Mechanics, 2007(04): 333-340.

[7]. LIU, Z.Q., et al., Study on Constitutive Equation of Metal Cutting Deformation. Tool Technology, 2008(03): 3-9.

[8]. Guo, Y.B., Q. Wen And M.F. Horstemeyer, An Internal State Variable Plasticity-Based Approach to Determine Dynamic Loading History Effects on Material Property in Manufacturing Processes. International Journal of Mechanical Sciences, 2005. 47(9): P. 1423-1441.

[9]. HU, X.T and SONG, Y.D, Contrastive Analysis of Three Dynamic Constitutive Models for TCTC4 Titanium Alloy. Ordnance Material Science and Engineering, 2013.

[10]. Q. Wen, Y.B. Guo and K.A. Woodbury., Adiabatic Shear Modeling and Its Influence on Machining Simulations: BCJ Vs. JC Model. ASME International Mechanical Engineering Congress and Exposition, 2005.

[11]. Scherzinger, W.M. And D.C. Hammerand, Constitutive Models in LAME. Sandia Report, 2007.

[12]. Johnson, G.R. And W.H. Cook, Fracture Characteristics of Three Metals Subjected to Various Strains, Strain Rates, Temperatures and Pressures. Engineering Fracture Mechanics, 1985. No. I(Vol. 21): P. Pp. 31-48.

[13]. LIU, H.W., Study on Dynamic Mechanical Properties and Damage Evolution of High Purity Aluminum, 2010, University of Ningbo.

[14]. LIU, X.Y., et al., Study on Constitutive Equation of Pure Al (L_2) High Temperature. Journal of Central South University of Technology, 1997(02): 57-60.

[15]. LI, X.Q. and LI, Y.B., Thermal Mechanical Coupling Analysis of Continuous Casting and Rolling Based On MARC Platform. China Mechanical Engineering, 2006.

[16]. Zhou, M. And M.P. Clode, A Constitutive Model and Its Identification For The Deformation Characterized By Dynamic Recovery. Transactions of the ASME, 1997.

[17]. Cram, D.G., et al., Modelling Discontinuous Dynamic Recrystallization Using A Physically Based Model For Nucleation. Acta Materialia, 2009. 57(17): P. 5218-5228.

[18]. CHEN, S.P. and S. VAN DER ZWAAG, Modeling Recrystallization Kinetics in AA1050 Following Simulated Breakdown Rolling. Metallurgical and Materials Transactions A, 2003.

[19]. Bammann, D.J., An Internal Varible Model of Viscoplastcity.1984

[20]. Salehghaffari, S., et al., A New Approach for Determination of Material Constants of Internal State Variable Based Plasticity Models and Their Uncertainty Quantification. Computational Materials Science, 2012. 55: P. 237-244.

[21]. Jordon, J.B., et al., Damage and Stress State Influence on the Bauschinger Effect in Aluminum Alloys. Mechanics of Materials, 2007. 39(10): P. 920-931. 